\title{
The Structure and Uniqueness of Generalized Solutions of the Spherically Symmetric Einstein-Scalar Equations ${ }^{\star}$
}

\author{
Demetrios Christodoulou* \\ Departments of Mathematics and Physics, Syracuse University, Syracuse, NY 13210, USA
}

\begin{abstract}
In a previous paper we proved the global existence of generalized solutions of the spherically symmetric Einstein-scalar field equations in the large. In this paper we study the regularity properties of the spacetime and the scalar field corresponding to a generalized solution. We also prove a uniqueness theorem which shows that a generalized solution is an extension of a classical solution.
\end{abstract}

\section{Section 0. Introduction}

In [1] we began the study of the global initial value problem for the Einsteinscalar equations $R_{\mu \nu}=8 \pi \partial_{\mu} \phi \partial_{\nu} \phi$ in the spherically symmetric case. In terms of a retarded time coordinate $u$ and a radial coordinate $r$, the spacetime metric has the form

$$
d s^{2}=-e^{2 v} d u^{2}-2 e^{v+\lambda} d u d r+r^{2} d \Sigma^{2},
$$

where $d \Sigma^{2}$ is the metric of the standard 2-sphere. The problem is best formulated in terms of the function $h:=\partial(r \phi) / \partial r$. If $f$ is a function of $u$ and $r$ we denote by $\bar{f}$ the mean value function of $f$ :

Defining then

$$
\bar{f}(u, r):=\frac{1}{r} \int_{0}^{r} f\left(u, r^{\prime}\right) d r^{\prime}
$$

$$
g:=\exp \left[-4 \pi \int_{r}^{\infty}(h-\bar{h})^{2} \frac{d r}{r}\right], \quad D:=\frac{\partial}{\partial u}-\frac{1}{2} \bar{g} \frac{\partial}{\partial r}
$$

\footnotetext{
* Research supported in part by National Science Foundation grants MCS-8201599 to the Courant Institute and PHY-8318350 to Syracuse University
} 
and

$$
m:=\frac{r}{2}\left(1-\frac{\bar{g}}{g}\right), \quad \xi:=2 r D \bar{h},
$$

we showed in [1] that the spherically symmetric Einstein-scalar equations are, through the identification $e^{v+\lambda}:=g, e^{v-\lambda}:=\bar{g}$ and $\phi:=\bar{h}$, equivalent to the equations

$$
D h=\frac{1}{2 r}(g-\bar{g})(h-\bar{h}) \text { and } \quad D m=-\frac{\pi}{g} \xi^{2} .
$$

The function $m(u, r)$ represents physically the mass which at retarded time $u$ is enclosed within the sphere of radius $r$. The initial data of the problem is the specification of the function $h$ at $u=0$, namely on a future light cone with vertex at the center of symmetry. The integral curves of $D$ play an important role in the problem and we call them characteristics.

In [1] we first proved the local, in retarded time, existence (Theorem 1 of [1]) and global uniqueness (Theorem 2 of [1]) of classical solutions of the initial value problem. We then proved that if the initial data is sufficiently small there exists a global classical solution (Theorem 3 of [1]). In [2] we studied the problem in the large. We introduced the concept of a generalized solution of our problem and we proved, without any restriction on the size of the initial data, the global existence of generalized solutions (Theorem 1 of [2]).

In the present paper we study first the regularity properties of the spacetime and the scalar field corresponding to a generalized solution (Sect. 1). Here the function $\xi$ plays a central role. This function is in physical terms the local radiative amplitude. We also study the behaviour of generalized solutions at null infinity (Sect. 2). We then state and prove the following uniqueness theorem (Theorem 1): a generalized solution having the same data as a classical solution coincides with it in the domain of existence of the latter. The proof of this theorem uses the quantity $L$, introduced in Sect. 3, which is a measure of the difference between the two solutions. In Sect. 4 we derive various estimates of which the main one depends on the spacetime integral of the main integral identity of [2] and on an inequality in Orlicz spaces. These estimates lead to an ordinary differential inequality which in turn leads to the proof of Theorem 1.

We wish to note that for generalized solutions of the Navier-Stokes equations a uniqueness theorem of the above type was proven by $J$. Leray in his original work of 1934 [3]. Despite the fact that we now have a better understanding of the regularity properties of those solutions (see [4]), the situation regarding uniqueness has remained unchanged.

In a subsequent paper we shall study the asymptotic behaviour of generalized solutions as $u \rightarrow \infty$. 


\section{Section 1. The Structure of Generalized Solutions}

We have seen in the proof of Theorem 1 of [2] that the restriction of $\xi$ to the characteristic $\chi_{u_{1}}\left(\cdot ; r_{1}\right)$ through each $\left(u_{1}, r_{1}\right), r_{1} \neq 0$ belongs to $L^{2}\left(0, u_{1}\right)$ considered as a function of $u$. This implies that the restrictions of $\bar{h}$ and $g$ to each $\chi_{u_{1}}\left(\cdot ; r_{1}\right)$ belong to $H_{1}\left(0, u_{1}\right)$ and the restrictions of $m$ and $\bar{g}$ are absolutely continuous functions of $u$. Hence the tangent of each characteristic is absolutely continuous in the parameter $u$. These results follow from the corresponding evolution laws.

In the course of the proof of Theorem 1 of [2] we have also seen that the metric functions $g$ and $\bar{g}$ are continuous with respect to $r$ even at $r=0$. However, this property is not enough to guarantee either uniqueness or existence of characteristics through a point on the central line $r=0$. Nevertheless, since, $g(u, 0)$ is a measurable function of $u$ and $0 \leqq g(u, 0) \leqq 1$, we can define the measure $g(u, 0) d u$ which represents proper time duration on the central line. We note that the function $g(u, 0)$ may vanish for a set of values of $u$ of non-zero $d u$ measure. Such a set will correspond to a set of events on the central line whose duration as observed from infinity is nonzero but whose proper time duration is zero.

Let us remind ourselves of the fact that the lines $u=$ const represent future light cones. If at a certain value of $u g^{1 / 2} \xi / \bar{g} r^{1 / 2} \in L^{2}\left(0, r_{0}\right)$ for some (and therefore for all) $r_{0}>0$, we shall call the corresponding light cone "regular." On the other hand, if at a certain value of $u$ the contrary is true, the corresponding light cone shall be called "singular." $\xi$ is physically the local radiative amplitude. By the definition of a generalized solution $g^{1 / 2} \xi / \bar{g} r^{1 / 2} \in L^{2}\left(\left(0, u_{0}\right) \times\left(0, r_{0}\right)\right), u_{0}, r_{0}$ arbitrary. Therefore at almost all values of $u g^{1 / 2} \xi / \bar{g} r^{1 / 2} \in L^{2}\left(0, r_{0}\right), r_{0}$ arbitrary. Thus we have:

Corollary 1. The set of singular cones is of zero measure.

From Proposition 2 of [2] we have:

Corollary 2. On a regular cone $\xi / \bar{g}^{1 / 2}$ is continuous and uniformly bounded and tends to 0 as $r \rightarrow 0$.

Corollary 3. On a regular cone $D m \rightarrow 0$ as $r \rightarrow 0$.

Let us consider the behaviour of the sectional curvatures. The nonvanishing components of the curvature tensor are given by (4.8)-(4.12) of [1]. The sectional curvatures for planes tangential to the light cones, that is $K\left(l, \zeta_{1}\right), K\left(l, \zeta_{2}\right)$ and $K\left(\zeta_{1}, \zeta_{2}\right)$ :

$$
K\left(l, \zeta_{1}\right)=K\left(l, \zeta_{2}\right)=\frac{4 \pi}{r^{2}} \frac{\bar{g}}{g}(h-\bar{h})^{2}, \quad K\left(\zeta_{1}, \zeta_{2}\right)=\frac{2 m}{r^{2}}
$$

are continuous everywhere in the complement of the central line. The same is true for

$$
R\left(n, \zeta_{1}, l, \zeta_{1}\right)=R\left(n, \zeta_{2}, l, \zeta_{2}\right)=\frac{m}{r^{3}} .
$$

On the other hand, the sectional curvatures for planes transversal to the light cones, that is $K\left(n, \zeta_{1}\right), K\left(n, \zeta_{2}\right)$ and $K(n, l)$ :

$$
K\left(n, \zeta_{1}\right)=K\left(n, \zeta_{2}\right)=\frac{\pi \xi^{2}}{r^{2} g \bar{g}}, \quad K(n, l)=\frac{4 \pi}{r^{2} g}(h-\bar{h}) \xi-\frac{2 m}{r^{3}},
$$


while continuous on each regular cone (we are excluding the vertices), may in fact blow up at the singular cones. However, at each $r>0: K\left(n, \zeta_{1}\right), K\left(n, \zeta_{2}\right) \in L^{1}\left(0, u_{0}\right)$ and $K(n, l) \in L^{2}\left(0, u_{0}\right), u_{0}$ arbitrary, as functions of $u$.

The following proposition clarifies the relation of the evolution law of $\bar{h}$ to that of $h$.

Proposition 1. On each regular cone the limit

exists and equals $D \bar{h}$.

$$
\lim _{\varepsilon \rightarrow 0} \frac{1}{\varepsilon} \int_{0}^{\varepsilon} D\left(\frac{1}{r} \int_{\delta}^{r} h d r\right) d \delta
$$

Proof. From the nonlinear evolution equation we can derive the evolution law of

$$
\frac{1}{r} \int_{\delta}^{r} h d r
$$

for any given $\delta>0$. We have:

$$
\begin{aligned}
D\left(\frac{1}{r} \int_{\delta}^{r} h d r\right)= & \frac{1}{2} \bar{g}\left(\frac{1}{r^{2}} \int_{\delta}^{r} h d r-\frac{1}{r} h\right)+\frac{1}{r} \int_{\delta}^{r}\left(D h+\frac{1}{2} \bar{g} \frac{\partial h}{\partial r}\right) d r \\
= & \frac{1}{2} \frac{\bar{g}}{r^{2}}\left(\int_{0}^{r} h d r-\int_{0}^{\delta} h d r\right)-\frac{1}{2 r} \bar{g} h \\
& +\frac{1}{r} \int_{\delta}^{r}\left(\frac{1}{2} \frac{\partial \bar{g}}{\partial r} h+\frac{1}{2} \bar{g} \frac{\partial h}{\partial r}-\frac{1}{2} \frac{\partial \bar{g}}{\partial r} \bar{h}\right) d r \\
= & \frac{1}{2 r^{2}} \bar{g}(r \bar{h}-\delta \bar{h}(\delta))-\frac{1}{2 r} \bar{g}(\delta) h(\delta)-\frac{1}{2 r} \int_{\delta}^{r} \frac{\partial \bar{g}}{\partial r} \bar{h} d r \\
= & -\frac{1}{2 r^{2}} \bar{g} \cdot \delta \bar{h}(\delta)-\frac{1}{2 r} \bar{g}(\delta)(h(\delta)-\bar{h}(\delta))+\frac{1}{2 r} \int_{\delta}^{r} \bar{g}(h-\bar{h}) \frac{d r}{r} .
\end{aligned}
$$

Now, on each regular cone

$$
\lim _{\delta \rightarrow 0} \int_{\delta}^{r} \bar{g}(h-\bar{h}) \frac{d r}{r}=\xi .
$$

Also, by the bound (5.6) of [2] on $\bar{h}$,

$$
\lim _{\delta \rightarrow 0} \delta \bar{h}(\delta)=0 .
$$

Thus, we need only show that

$$
\lim _{\varepsilon \rightarrow 0}-\int_{\varepsilon}^{1} \bar{g}(\delta)(h(\delta)-\bar{h}(\delta)) d \delta=0 .
$$

But

$$
\begin{aligned}
\left|\int_{0}^{r} \bar{g}(h-\bar{h}) d r\right| \leqq \int_{0}^{r} g|h-\bar{h}| d r & \leqq\left(\int_{0}^{r} g r d r\right)^{1 / 2}\left(\int_{0}^{r} g(h-\bar{h})^{2} \frac{d r}{r}\right)^{1 / 2} \\
& \leqq\left(r^{2} / 2\right)^{1 / 2}\left(\frac{1}{4 \pi}(g(r)-g(0))\right)^{1 / 2}
\end{aligned}
$$


Therefore, by the continuity of $g$ with respect to $r$ at $r=0$,

$$
\frac{1}{\varepsilon}\left|\int_{0}^{\varepsilon} \bar{g}(\delta)(h(\delta)-\bar{h}(\delta)) d \delta\right| \leqq \frac{1}{(8 \pi)^{1 / 2}}(g(\varepsilon)-g(0))^{1 / 2} \rightarrow 0 \quad \text { as } \quad \varepsilon \rightarrow 0 .
$$

We shall now show

Proposition 2. On a regular cone $\bar{g}^{1 / 2} \bar{h}$ tends to a limit $f$ as $r \rightarrow 0$. The function $f(u)$, which is thus defined for almost all $u$, belongs to $L^{2}\left(0, u_{0}\right), u_{0}$ arbitrary.

Proof. Consider the identity

$$
\bar{g}\left(\bar{h}-\frac{\xi}{g}\right)^{2}+\int_{r}^{r_{0}}(g-\bar{g}) \bar{h}^{2} \frac{d r}{r}=\left[\bar{g}\left(\bar{h}-\frac{\xi}{g}\right)^{2}\right]_{r=r_{0}}+\int_{r}^{r_{0}} \frac{(g-\bar{g})}{\bar{g}^{2}} \xi^{2} \frac{d r}{r} .
$$

This identity shows that on a regular cone the integral

$$
\int_{\mathrm{r}}^{r_{0}}(g-\bar{g}) \bar{h}^{2} \frac{d r}{r}
$$

is bounded by a constant independent of either $r$ or $r_{0}$. Also, integrating (1.1) with respect to $u$ shows that the integral

$$
\int_{0}^{u_{0}} \int_{r}^{r_{0}}(g-\bar{g}) \bar{h}^{2} \frac{d r}{r} d u
$$

is bounded by a constant independent of either $r$ or $r_{0}$. It then follows by monotone convergence that $(g-\bar{g})^{1 / 2} \bar{h} / r^{1 / 2} \in L^{2}(0, \infty)$. The identity (1.1) then shows that on a regular cone the function $\bar{g}(\bar{h}-\xi / \bar{g})^{2}$ tends to the limit

$$
f^{2}:=\left[\bar{g}\left(\bar{h}-\frac{\xi}{g}\right)^{2}\right]_{r=r_{0}}+\int_{0}^{r_{0}} \frac{(g-\bar{g})}{\bar{g}^{2}} \xi^{2} \frac{d r}{r}-\int_{0}^{r_{0}}(g-\bar{g}) \bar{h}^{2} \frac{d r}{r}
$$

as $r \rightarrow 0$. Hence, considering the fact that on a regular cone $\bar{g}^{1 / 2}(\bar{h}-\xi / \bar{g})$ is continuous for $r>0$, the function $\bar{g}^{1 / 2}(\bar{h}-\xi / \bar{g})$ tends to a limit $f= \pm \sqrt{f^{2}}$ as $r \rightarrow 0$. Therefore, since on a regular cone $\xi / \bar{g}^{1 / 2} \rightarrow 0$ as $r \rightarrow 0, \bar{g}^{1 / 2} \bar{h} \rightarrow f$ as $r \rightarrow 0$. The function $f(u)$, defined in this way for almost all $u$, is then a measurable function of $u$. The identity (1.1) implies, in view of Proposition 2 of [2], that the functions $\left(\bar{g} \bar{h}^{2}\right)(\cdot, r)$, $r>0$, are dominated by an integrable function of $u$. By the dominated convergence theorem we then have $f \in L^{2}\left(0, u_{0}\right), u_{0}$ arbitrary, and

$$
\int_{0}^{u_{0}} f^{2}(u) d u=\lim _{r \rightarrow 0} \int_{0}^{u_{0}}\left(\bar{g} \bar{h}^{2}\right)(u, r) d u
$$

Corollary 4. For a regular cone $\bar{h}$ is continuous at the vertex if $g$ is positive there. Using Proposition 2 we can also show:

Proposition 3. For a generalized solution, the quantity

$$
\int_{0}^{\infty} h^{2}(u, r) d r
$$


is an absolutely continuous function of $u$ and

$$
\begin{aligned}
\frac{\partial}{\partial u}\left(\int_{0}^{\infty} h^{2}(u, r) d r\right) & +\frac{1}{2} \int_{0}^{\infty}\left[(g-\bar{g}) \bar{h}^{2}+\bar{g}(h-\bar{h})^{2}\right](u, r) \frac{d r}{r} \\
& +\frac{1}{2} f^{2}(u)=\frac{1}{8 \pi}(1-g(u, 0)) .
\end{aligned}
$$

\section{Section 2. The Null Infinity of Generalized Solutions}

Let us introduce the following notation. Given a function $f$ defined in $Q$, the complement of the central line, we shall write

$$
f=O_{k}\left(r^{-l}\right)
$$

if $f$ and $\partial^{i} f / \partial r^{i}, i=1 \ldots k$, are continuous in $Q$ and at each $u: f=O\left(r^{-l}\right)$ and $\partial^{i} f / \partial r^{i}=O\left(r^{-l-i}\right), i=1 \ldots k$, for $r \rightarrow \infty$. According to (5.2) and (5.4) we have

$$
h=O_{1}\left(r^{-3}\right) .
$$

Let us set

$$
N:=\int_{0}^{\infty} h d r
$$

Then, since

$$
\bar{h}=\frac{N}{r}-\frac{1}{r} \int_{0}^{\infty} h d r,
$$

and

$$
\int_{r}^{\infty} h d r=O_{2}\left(r^{-2}\right)
$$

we obtain:

$$
\bar{h}=\frac{N}{r}+O_{2}\left(r^{-3}\right) .
$$

It follows that

$$
g=1-2 \pi \frac{N^{2}}{r^{2}}+O_{2}\left(r^{-4}\right) .
$$

According to (4.7) of part I, the total mass $M$ can be expressed in the form

$$
M=\frac{1}{2} \int_{0}^{\infty}(1-g) d r
$$

Then, since

$$
1-\bar{g}=\frac{1}{r} \int_{0}^{r}(1-g) d r
$$


we have:

$$
1-\bar{g}=\frac{2 M}{r}-\frac{1}{r} \int_{r}^{\infty}(1-\bar{g}) d r .
$$

Taking into account (2.3) we obtain from (2.4) that:

$$
\bar{g}=1-\frac{2 M}{r}+2 \pi \frac{N^{2}}{r^{2}}+O_{3}\left(r^{-4}\right) .
$$

At each regular cone we can define

$$
\Xi:=\lim _{\delta \rightarrow 0} \int_{\delta}^{\infty} \bar{g}(h-\bar{h}) \frac{d r}{r} .
$$

The function $\Xi(u)$ is defined for almost all values of $u$. $\Xi$ is physically the total radiative amplitude. It follows from Proposition 2 of [2] that $\Xi \in L^{2}\left(0, u_{0}\right), u_{0}$ arbitrary. Since

$$
\xi=\Xi-\int_{r}^{\infty} \bar{g}(h-\bar{h}) \frac{d r}{r},
$$

taking into account (2.2) and (2.5) we obtain:

$$
\xi=\Xi+\frac{N}{r}-\frac{N M}{r^{2}}+O_{2}\left(r^{-3}\right) .
$$

We shall now find the relations between the functions $N(u), M(u)$ and $\Xi(u)$. Since

$$
\partial \hbar / \partial u=\frac{\partial N / \partial u}{r}-\frac{1}{r} \int_{r}^{\infty} \partial h / \partial u d r
$$

and, by the nonlinear evolution equation, $\partial h / \partial u=O\left(r^{-3}\right)$, we have

$$
\partial \bar{h} / \partial u=\frac{\partial N / \partial u}{r}+O\left(r^{-3}\right) .
$$

Since, furthermore, $\frac{1}{2} \bar{g} \partial \bar{h} / \partial r=O\left(r^{-2}\right)$, we obtain

$$
D \bar{h}=\frac{\partial N / \partial u}{r}+O\left(r^{-2}\right) .
$$

On the other hand, by (2.7),

$$
D \bar{h}=\frac{\xi}{2 r}=\frac{\Xi}{2 r}+O\left(r^{-2}\right) .
$$

Comparison of (2.8) with (2.9) yields:

$$
\frac{\partial N}{\partial u}=\frac{1}{2} \Xi
$$

Taking the limit of the mass flux relation [(5.43) of [2]] as $r_{1} \rightarrow \infty$ we obtain:

$$
M\left(u_{1}\right)+\pi \int_{0}^{u_{1}} \Xi^{2} d u=M_{0} .
$$


Therefore

$$
\frac{\partial M}{\partial u}=-\pi \Xi^{2}
$$

Also, the fact that $M(u)$ tends to a nonnegative limit $M_{1}$ as $u \rightarrow \infty$ implies that $\Xi \in L^{2}(0, \infty)$. We then conclude from (2.10) and (2.12) that $N \in H_{1}\left(0, u_{0}\right), u_{0}$ arbitrary, and $M(u)$ is an absolutely continuous function of $u$. The function $\pi \Xi^{2}(u)$ represents the radiative power at retarded time $u$. It may blow up at the values of $u$ corresponding to the singular cones. However, given any $\varepsilon>0$ there exists a $\delta>0$ such that for all systems of retarded time intervals whose total duration is less than $\delta$, the total energy radiated in these intervals is less than $\varepsilon$.

From the expressions for the sectional curvatures given in the preceding section we can obtain, using (2.2), (2.3), (2.5) and (2.7), their behaviour at null infinity. We list below the leading terms:

$$
\begin{aligned}
K\left(n, \zeta_{1}\right) & =K\left(n, \zeta_{2}\right)=\frac{\pi \Xi^{2}}{r^{2}}+\cdots, \\
K(n, l) & =-\frac{(4 \pi N \Xi+2 M)}{r^{3}}+\cdots, \\
R\left(N, \zeta_{1}, l, \zeta_{1}\right) & =R\left(n, \zeta_{2}, l, \zeta_{2}\right)=\frac{M}{r^{3}}+\cdots, \\
K\left(\zeta_{1}, \zeta_{2}\right) & =\frac{2 M}{r^{3}}+\cdots, \\
K\left(l, \zeta_{1}\right) & =K\left(l, \zeta_{2}\right)=\frac{4 \pi N^{2}}{r^{4}}+\cdots
\end{aligned}
$$

\section{Section 3. The Uniqueness Theorem}

The purpose of this and the last section is the proof of:

Theorem 1. A generalized solution having the same data as a classical solution coincides with it in the domain of existence of the latter.

According to the above theorem a generalized solution is an extension of a classical solution. Let $h$ be a generalized solution and $h_{0}$ be a classical solution having the same data

$$
h(0, r)=h_{0}(0, r)
$$

In this and the last section all quantities subscripted by a 0 shall refer to the classical solution. The generalized solution is a global solution, while the classical solution will in general have maximal interval of existence $\left[0, u_{1}[\right.$. We shall show that in any closed subinterval $\left[0, u_{0}\right]$ of the interval $\left[0, u_{1}\left[h\right.\right.$ coincides with $h_{0}$. We therefore confine our attention in the following to a given subinterval $\left[0, u_{0}\right]$.

Let:

$$
\beta:=h-h_{0},
$$




$$
B:=\int_{r}^{\infty}(\beta-\bar{\beta})^{2} \frac{d r}{r}
$$

and

$$
\alpha:=e^{-4 \pi B}
$$

We have

$$
g / \alpha=e^{\psi} g_{0}
$$

where

$$
\psi:=-8 \pi \int_{r}^{\infty}(\beta-\bar{\beta})\left(h_{0}-\bar{h}_{0}\right) \frac{d r}{r} .
$$

By Theorem 1, of [2] at each $u: h-\bar{h} \in L^{2}(0, \infty)$, and

$$
\int_{0}^{\infty}(h-\bar{h})^{2} d r
$$

is uniformly bounded in $\left[0, u_{0}\right]$. It follows that the same is true for $\beta-\bar{\beta}$. In particular, at each $u ; \beta-\bar{\beta} \in L^{1}(0,1)$ and

$$
\int_{0}^{1}|\beta-\bar{\beta}| d r
$$

is uniformly bounded. Then, since $\partial \bar{h}_{0} / \partial r$ is uniformly bounded, $h_{0}$ being a classical solution in $\left[0, u_{1}\left[\times\left[0, \infty\left[\supset\left[0, u_{0}\right] \times[0, \infty[\right.\right.\right.\right.$, the integral

$$
\int_{0}^{1}|\beta-\bar{\beta}|\left|\partial \bar{h}_{0} / \partial r\right| d r
$$

is bounded by a constant. For any given $r_{0}>0$,

$$
\sup _{r \geqq r_{0}}\{r|h-\bar{h}|\}
$$

is uniformly bounded in $\left[0, u_{0}\right]$. Hence

$$
\sup _{r \geqq 1}\{r|\beta-\bar{\beta}|\}
$$

is bounded by a constant, and since furthermore $r^{2} \partial \bar{h}_{0} / \partial r$ is uniformly bounded, the integral

$$
\int_{1}^{\infty}|\beta-\bar{\beta}|\left|\partial \bar{h}_{0} / \partial r\right| d r
$$

is bounded by a constant. Therefore

$$
\int_{0}^{\infty}|\beta-\bar{\beta}|\left|\partial \bar{h}_{0} / \partial r\right| d r
$$

is bounded by a constant. Hence

$$
|\psi| \leqq 8 \pi \int_{0}^{\infty}|\beta-\bar{\beta}|\left|\partial \bar{h}_{0} / \partial r\right| d r \leqq C .
$$


Here and in the following $C$ denotes various positive constants (which may depend on $u_{0}$ ). Since $h_{0}$ is a classical solution, $g_{0}$ is bounded from below by a positive constant. We thus conclude from (8.4) that there exists a constant $k>1$ such that

$$
\frac{1}{k} \leqq \frac{g}{\alpha} \leqq k, \quad \text { and therefore also } \quad \frac{1}{k} \leqq \frac{\bar{g}}{\alpha} \leqq k .
$$

We now define the nonnegative quantities

and

$$
N:=\int_{0}^{\infty}(1-\alpha) d r
$$

$$
L:=\int_{0}^{\infty} \frac{g}{\alpha}(1-\alpha) d r
$$

The quantities $N$ and $L$ are measures of the difference between the two solutions $h$ and $h_{0}$ at each value of $u$. If we were to set $h_{0}=0$ in $N$ and $L$ we would obtain $N=L=2 M$, where $M$ is the total mass corresponding to $h$. By (8.7) we have:

$$
\frac{N}{k} \leqq L \leqq k N
$$

The fact that at each $u(1-\alpha)=O\left(r^{-2}\right)$ as $r \rightarrow \infty$, implies that

$$
\int_{0}^{\infty} \frac{\bar{\alpha}}{\alpha}(\beta-\bar{\beta})^{2} d r=\frac{1}{4 \pi} \int_{0}^{\infty} r \bar{\alpha} \frac{\partial}{\partial r}\left(\frac{1}{\alpha}-1\right)=\frac{1}{4 \pi} \int_{0}^{\infty}\left(\frac{1}{\alpha}-1\right) d(r \bar{\alpha})=\frac{1}{4 \pi} \int_{0}^{\infty}(1-\alpha) d r .
$$

Thus $N$ can also be expressed in the form:

$$
N=4 \pi \int_{0}^{\infty} \frac{\bar{\alpha}}{\alpha}(\beta-\bar{\beta})^{2} d r
$$

Using this expression together with the corresponding expressions

$$
M[h]=2 \pi \int_{0}^{\infty} \frac{\bar{g}}{g}(h-\bar{h})^{2} d r, \quad M\left[h_{0}\right]=2 \pi \int_{0}^{\infty} \frac{\bar{g}_{0}}{g_{0}}\left(h_{0}-\bar{h}_{0}\right)^{2} d r
$$

for the total mass corresponding to $h$ and $h_{0}$ respectively, and taking into account (3.7), we can show that $N$, and therefore by (3.10) also $L$, is bounded by a constant multiple of the initial total mass:

$$
N, L \leqq C M_{0}
$$

Our proof of Theorem 1 is by deriving an ordinary differential inequality for the quantity $L$.

Subtracting the nonlinear evolution equation for $h_{0}$ from that for $h$ we obtain

$$
D \beta-\frac{1}{2 r}(g-\bar{g})(\beta-\bar{\beta})=\frac{1}{2}\left(\bar{g}-\bar{g}_{0}\right) \frac{\partial h_{0}}{\partial r}+\frac{1}{2 r}\left(g-g_{0}-\bar{g}+\bar{g}_{0}\right)\left(h_{0}-\bar{h}_{0}\right)
$$

where

$$
D:=\frac{\partial}{\partial u}-\frac{1}{2} \bar{g} \frac{\partial}{\partial r}
$$


is the derivative along the characteristics of the generalized solution. Subtracting also the evolution equation for $\bar{h}_{0}$ from that for $\bar{h}$ we obtain:

$$
D \bar{\beta}-\frac{\theta}{2 r}=\frac{\eta}{2 r}+\frac{1}{2}\left(\bar{g}-\bar{g}_{0}\right) \frac{\partial \bar{h}_{0}}{\partial r} \text {. }
$$

Here

$$
\theta:=\lim _{\delta \rightarrow 0} \int_{\delta}^{r} \bar{g}(\beta-\bar{\beta}) \frac{d r}{r} \quad(\text { defined for almost all } u)
$$

and

$$
\eta:=\int_{0}^{r}\left(\bar{g}-\bar{g}_{0}\right)\left(h_{0}-\bar{h}_{0}\right) \frac{d r}{r} .
$$

We have $\theta=\xi-\zeta$, where

$$
\zeta=\int_{0}^{r} \bar{g}\left(h_{0}-\bar{h}_{0}\right) \frac{d r}{r}
$$

since

$$
\frac{|\zeta|}{\bar{g}} \leqq \int_{0}^{r}\left|\frac{\partial \bar{h}_{0}}{\partial r}\right| d r
$$

The fact that $\partial \bar{h}_{0} / \partial r$ and $r^{2} \partial \bar{h}_{0} / \partial r$ are uniformly bounded implies that $\zeta / \bar{g} r$ and $\zeta / \bar{g}$ are uniformly bounded. By Theorem 1 of [2], $g^{1 / 2} \xi / \bar{g} r^{1 / 2} \in L^{2}\left(\left(0, u_{0}\right) \times\left(0, r_{0}\right)\right), r_{0}$ arbitrary, and (by Proposition 2 of [2])

$$
\sup _{r \geqq 0}\left|\left(\xi / \bar{g}^{1 / 2}\right)(u, r)\right| \in L^{2}\left(0, u_{0}\right),
$$

and at almost all $u \xi / \bar{g}^{1 / 2} \rightarrow 0$ as $r \rightarrow 0$. It follows that also $g^{1 / 2} \theta / \bar{g} r^{1 / 2} \in L^{2}\left(\left(0, u_{0}\right)\right.$ $\left.\times\left(0, r_{0}\right)\right), r_{0}$ arbitrary, and

$$
\sup _{r \geqq 0}\left|\left(\theta / \bar{g}^{1 / 2}\right)(u, r)\right| \in L^{2}\left(0, u_{0}\right),
$$

and at almost all $u \theta / \bar{g}^{1 / 2} \rightarrow 0$ as $r \rightarrow 0$.

The function $B$ defined by (3.2) is weakly differentiable in the complement of the central line. By (3.13) and (3.14) we have:

$$
\begin{aligned}
D B= & \frac{1}{2 r} \bar{g}(\beta-\bar{\beta})^{2}+\int_{r}^{\infty} 2(\beta-\bar{\beta})\left[D \beta+\frac{1}{2} \bar{g} \frac{\partial \beta}{\partial r}-\frac{1}{2} \bar{g} \frac{\partial \bar{\beta}}{\partial r}-D \bar{\beta}\right] \frac{d r}{r} \\
= & \frac{1}{2 r} \bar{g}(\beta-\bar{\beta})^{2}+\int_{r}^{\infty}(\beta-\bar{\beta})\left[\frac{\partial g}{\partial r}(\beta-\bar{\beta})+\bar{g} \frac{\partial \beta}{\partial r}\right. \\
& -\bar{g} \frac{(\beta-\bar{\beta})}{r}-\frac{\theta}{r}+\left(\bar{g}-\bar{g}_{0}\right) \frac{\partial h_{0}}{\partial r} \\
& \left.+\left(g-g_{0}-\bar{g}+\bar{g}_{0}\right) \frac{\left(h_{0}-\bar{h}_{0}\right)}{r}-\frac{\eta}{r}-\left(\bar{g}-\bar{g}_{0}\right) \frac{\partial \bar{h}_{0}}{\partial r}\right] \frac{d r}{r} .
\end{aligned}
$$


Taking into account the fact that

$$
\frac{1 \partial \bar{g}}{r} \frac{\partial}{\partial r}(\beta-\bar{\beta})^{2}+\bar{g} \frac{(\beta-\bar{\beta}) \partial \beta}{r}-\bar{g} \frac{(\beta-\bar{\beta})^{2}}{r^{2}}=\frac{g}{2 r^{2}}(\beta-\bar{\beta})^{2}+\frac{\partial}{\partial r}\left[\frac{\bar{g}(\beta-\bar{\beta})^{2}}{2 r}\right],
$$

we obtain

$$
D B=\int_{r}^{\infty}(\beta-\bar{\beta})\left[\frac{g}{2}(\beta-\bar{\beta})-\theta\right] \frac{d r}{r^{2}}+\int_{r}^{\infty}(\beta-\bar{\beta}) \omega \frac{d r}{r}
$$

where

$$
\omega:=\left(g-g_{0}\right) \frac{\partial \bar{h}_{0}}{\partial r}+\left(\bar{g}-\bar{g}_{0}\right)\left(\frac{\partial h_{0}}{\partial r}-2 \frac{\partial \bar{h}_{0}}{\partial r}\right)-\frac{\eta}{r} .
$$

We shall now show:

Lemma 1. At almost all $u$

$$
\delta D B(u, \delta) \rightarrow 0 \quad \text { as } \quad \delta \rightarrow 0 .
$$

Proof. The function $\omega$, defined above, is uniformly bounded; hence the second integral in (3.17) is bounded by a constant multiple of

$$
\int_{r}^{\infty}|\beta-\bar{\beta}| \frac{d r}{r}
$$

Since at each $u(\beta-\bar{\beta}) \in L^{1}(0,1)$, we have:

$$
\delta \int_{\delta}^{\delta^{1 / 2}}|\beta-\bar{\beta}| \frac{d r}{r} \leqq \int_{\delta}^{\delta^{1 / 2}}|\beta-\bar{\beta}| d r \rightarrow 0 \quad \text { as } \quad \delta \rightarrow 0,
$$

and

$$
\delta \int_{\delta^{1 / 2}}^{1}|\beta-\bar{\beta}| \frac{d r}{r} \leqq \delta^{1 / 2} \int_{0}^{1}|\beta-\bar{\beta}| d r \rightarrow 0 \quad \text { as } \quad \delta \rightarrow 0
$$

Therefore

$$
\delta \int_{\delta}^{1}|\beta-\bar{\beta}| \frac{d r}{r} \rightarrow 0 \quad \text { as } \quad \delta \rightarrow 0
$$

Since

$$
\sup _{r \geqq 1}\{r|\beta-\bar{\beta}|\}
$$

is bounded by a constant, the integral

$$
\int_{1}^{\infty}|\beta-\bar{\beta}| \frac{d r}{r}
$$

is bounded by a constant. We conclude that at all $u$,

$$
\delta \int_{\delta}^{\infty}|\beta-\bar{\beta}| \frac{d r}{r} \rightarrow 0 \quad \text { as } \quad \delta \rightarrow 0 .
$$


The first integral in (3.17) is bounded by $\frac{1}{2} J_{1}+J_{2}$, where

and

$$
J_{1}:=\int_{r}^{\infty} g(\beta-\bar{\beta})^{2} \frac{d r}{r^{2}}
$$

$$
J_{2}:=\int_{r}^{\infty}|\beta-\bar{\beta}||\theta| \frac{d r}{r^{2}}
$$

By (3.7) we have

$$
J_{1} \leqq k \int_{r}^{\infty} \alpha(\beta-\bar{\beta})^{2} \frac{d r}{r^{2}}=\frac{k}{4 \pi} \int_{r}^{\infty} \frac{\partial \alpha}{\partial r} \frac{d r}{r}
$$

Since $\alpha$ is continuous with respect to $r$ at $r=0$, we have

$$
\delta \int_{\delta}^{\delta^{1 / 2}} \frac{\partial \alpha}{\partial r} \frac{d r}{r} \leqq \int_{\delta}^{\delta^{1 / 2}} \frac{\partial \alpha}{\partial r} d r=\alpha\left(\delta^{1 / 2}\right)-\alpha(\delta) \rightarrow 0 \quad \text { as } \quad \delta \rightarrow 0 .
$$

Also,

$$
\delta \int_{\delta^{1 / 2}}^{\infty} \frac{\partial \alpha}{\partial r} \frac{d r}{r} \leqq \delta^{1 / 2} \int_{0}^{\infty} \frac{\partial \alpha}{\partial r} d r=\delta^{1 / 2}(1-\alpha(0)) \rightarrow 0 \quad \text { as } \quad \delta \rightarrow 0 .
$$

We conclude that at all $u$,

$$
\delta J_{1}(u, \delta) \rightarrow 0 \quad \text { as } \quad \delta \rightarrow 0 .
$$

We decompose $J_{2}(u, \delta)$ into:

$$
J_{2}(\delta)=\int_{\delta}^{\delta^{1 / 2}}|\beta-\bar{\beta}||\theta| \frac{d r}{r^{2}}+\int_{\delta^{1 / 2}}^{1}|\beta-\bar{\beta}||\theta| \frac{d r}{r^{2}}+\int_{1}^{\infty}|\beta-\bar{\beta}||\theta| \frac{d r}{r^{2}} .
$$

Since $\theta / g^{1 / 2} r^{1 / 2} \in L^{2}\left(\left(0, u_{0}\right) \times(0,1)\right)$, at almost all $u$ we have $\theta / g^{1 / 2} r^{1 / 2} \in L^{2}(0,1)$. Thus, taking into account (3.7) we can estimate:

$$
\begin{aligned}
& \delta \int_{\delta}^{\delta^{1 / 2}}|\beta-\bar{\beta}||\theta| \frac{d r}{r^{2}} \leqq \int_{\delta}^{\delta^{1 / 2}}|\beta-\bar{\beta}||\theta| \frac{d r}{r} \\
& \leqq\left(\int_{\delta}^{\delta^{1 / 2}} g(\beta-\bar{\beta})^{2} \frac{d r}{r}\right)^{1 / 2}\left(\int_{\delta}^{\delta^{1 / 2}} \frac{\theta^{2}}{g} \frac{d r}{r}\right)^{1 / 2} \\
& \leqq k^{1 / 2}\left(\alpha\left(\delta^{1 / 2}\right)-\alpha(\delta)\right)^{1 / 2}\left(\int_{\delta}^{\delta^{1 / 2}} \frac{\theta^{2}}{g} \frac{d r}{r}\right)^{1 / 2} \rightarrow 0 \text { as } \delta \rightarrow 0,
\end{aligned}
$$

and

$$
\begin{gathered}
\delta \int_{\delta^{1 / 2}}^{1}|\beta-\bar{\beta}||\theta| \frac{d r}{r^{2}} \leqq \delta^{1 / 2} \int_{0}^{1}|\beta-\bar{\beta}||\theta| \frac{d r}{r} \\
\leqq \delta^{1 / 2}\left(\int_{0}^{1} g(\beta-\bar{\beta})^{2}\right)^{1 / 2}\left(\int_{0}^{1} \frac{\theta^{2}}{g} \frac{d r}{r}\right)^{1 / 2} \\
\leqq \delta^{1 / 2} k^{1 / 2}\left(\int_{0}^{1} \frac{\theta^{2}}{g} \frac{d r}{r}\right)^{1 / 2} \rightarrow 0 \text { as } \delta \rightarrow 0 .
\end{gathered}
$$


Furthermore, since the integral

$$
\int_{1}^{\infty}|\beta-\bar{\beta}| \frac{d r}{r^{2}}
$$

is bounded by a constant, the third integral in (3.19) is bounded by a constant multiple of

$$
\sup _{r}|\theta|,
$$

which is finite for almost all $u$. We conclude that at almost all $u$.

$$
\delta J_{2}(u, \delta) \rightarrow 0 \quad \text { as } \quad \delta \rightarrow 0 .
$$

The Lemma then follows.

The quantity $L$ defined by (3.9) is a weakly differentiable function of $u$ and

$$
\frac{\partial L}{\partial u}=I_{1}+I_{2}
$$

where

$$
I_{1}=-\int_{0}^{\infty} \frac{g}{\alpha} \frac{\partial \alpha}{\partial u} d r=4 \pi \int_{0}^{\infty} g \frac{\partial B}{\partial u} d r
$$

and (see (3.4)):

$$
I_{2}=\int_{0}^{\infty}(1-\alpha) \frac{\partial}{\partial u}(g / \alpha) d r=\int_{0}^{\infty}(1-\alpha) e^{\psi}\left(g_{0} \frac{\partial \psi}{\partial u}+\frac{\partial g_{0}}{\partial u}\right) d r .
$$

Now, Lemma 1, together with the fact that at almost all $u D B=O\left(r^{-2}\right)$ as $r \rightarrow \infty$, implies that:

$$
\int_{0}^{\infty} D B g d r=\int_{0}^{\infty} D B d(r \bar{g})=-\int_{0}^{\infty} r \bar{g} \frac{\partial D B}{\partial r} d r
$$

Therefore:

$$
I_{1}=4 \pi \int_{0}^{\infty}\left(D B+\frac{1}{2} \bar{g} \frac{\partial B}{\partial r}\right) g d r=-4 \pi \int_{0}^{\infty} \bar{g}\left(r \frac{\partial D B}{\partial r}-\frac{1}{2} g \frac{\partial B}{\partial r}\right) d r .
$$

From (3.17) we have:

$$
\bar{g}\left(r \frac{\partial D B}{\partial r}-\frac{1}{2} g \frac{\partial B}{\partial r}\right)=\bar{g} \frac{(\beta-\bar{\beta})}{r} \theta-\bar{g}(\beta-\bar{\beta}) \omega .
$$

Substituting this in (3.22) and considering the fact that

$$
\bar{g} \frac{(\beta-\bar{\beta})}{r} \theta=\theta \frac{\partial \theta}{\partial r}=\frac{\partial}{\partial r}\left(\frac{1}{2} \theta^{2}\right)
$$

and that at almost all $u: \theta \rightarrow 0$ as $r \rightarrow 0$, we obtain:

$$
I_{1}=-2 \pi \Theta^{2}+4 \pi \int_{0}^{\infty} \bar{g}(\beta-\bar{\beta}) \omega d r
$$


where

$$
\Theta(u):=\lim _{r \rightarrow \infty} \theta(u, r) ; \quad \Theta \in L^{2}\left(0, u_{0}\right) .
$$

Also, from (3.5), (3.13) and (3.14) we obtain:

$$
\begin{aligned}
\frac{\partial \psi}{\partial u} & =-8 \pi \int_{r}^{\infty}\left[(\beta-\bar{\beta}) \frac{\partial^{2} \bar{h}_{0}}{\partial u \partial r}+\frac{\partial \hbar_{0}}{\partial r}\left(\frac{\partial \beta}{\partial u}-\frac{\partial \bar{\beta}}{\partial u}\right)\right] d r \\
& =-8 \pi \int_{r}^{\infty}\left\{(\beta-\bar{\beta}) \frac{\partial^{2} \bar{h}_{0}}{\partial u \partial r}+\frac{1}{2} \frac{\partial \hbar_{0}}{\partial r}\left[\frac{\partial}{\partial r}(\bar{g}(\beta-\bar{\beta}))-\frac{\theta}{r}+\omega\right]\right\} d r,
\end{aligned}
$$

that is:

$$
\begin{aligned}
\frac{\partial \psi}{\partial u}= & 4 \pi \frac{\partial \bar{h}_{0}}{\partial r} \bar{g}(\beta-\bar{\beta})+4 \pi \int_{r}^{\infty} \frac{\partial \bar{h}_{0}}{\partial r} \frac{\theta}{r} d r \\
& -8 \pi \int_{r}^{\infty}\left[(\beta-\bar{\beta}) \mu+\frac{1}{2} \frac{\partial \bar{h}_{0}}{\partial r} \omega\right] d r
\end{aligned}
$$

where

$$
\mu:=\frac{\partial^{2} \bar{h}_{0}}{\partial u \partial r}-\frac{1}{2} \bar{g} \frac{\partial^{2} \bar{h}_{0}}{\partial r^{2}}
$$

\section{Section 4. The Orlicz Inequality; Proof of Theorem 2}

To derive an ordinary differential inequality for $L$, we have to estimate $\partial L / \partial u$, given by (3.20), in terms of $L$. In estimating $I_{1}$ we shall use the following:

Lemma 2. There is a universal constant $c$ such that

$$
\left|g-g_{0}\right| \leqq c(1-\alpha)^{1 / 2}
$$

Proof. Let

$$
h_{t}:=h_{0}+t \beta, \quad 0 \leqq t \leqq 1,
$$

and let

$$
A(t):=A\left[h_{t}\right]=\int_{r}^{\infty}\left(h_{t}-\bar{h}_{t}\right)^{2} \frac{d r}{r} .
$$

Then $h_{1}=h$ and

$$
g-g_{0}=e^{-4 \pi A(1)}-e^{-4 \pi A(0)}=\int_{0}^{1} \frac{\partial}{\partial t}\left(e^{-4 \pi A(t)}\right) d t,
$$

since

$$
\frac{\partial A(t)}{\partial t}=2 \int_{r}^{\infty}\left(h_{t}-\bar{h}_{t}\right)(\beta-\bar{\beta}) \frac{d r}{r}
$$


we have

$$
g-g_{0}=-8 \pi \int_{0}^{1}\left(\int_{r}^{\infty}\left(h_{t}-\bar{h}_{t}\right)(\beta-\bar{\beta}) \frac{d r}{r}\right) e^{-4 \pi A(t)} d t .
$$

Therefore (by the Schwarz inequality)

$$
\left|g-g_{0}\right| \leqq 8 \pi\left(\int_{r}^{\infty}(\beta-\bar{\beta})^{2} \frac{d r}{r}\right)^{1 / 2} \int_{0}^{1} A^{1 / 2}(t) e^{-4 \pi A(t)} d t=8 \pi b \cdot B^{1 / 2}
$$

where

$$
b:=\max _{x \in[0 \infty]}\left\{x^{1 / 2} e^{-4 \pi x}\right\} .
$$

On the other hand, $\left|g-g_{0}\right| \leqq 1$. Hence:

$$
\left|g-g_{0}\right| \leqq \min \left\{8 \pi b B^{1 / 2}, 1\right\} \leqq\left(\frac{1-e^{-4 \pi B}}{1-e^{-1 / 16 \pi b^{2}}}\right)^{1 / 2}
$$

According to (3.24),

$$
I_{1} \leqq 4 \pi\left|\int_{0}^{\infty} \bar{g}(\beta-\bar{\beta}) \omega d r\right|
$$

and form (3.18) we obtain

$$
\begin{aligned}
\left|\int_{0}^{\infty} \bar{g}(\beta-\bar{\beta}) \omega d r\right| \leqq & \int_{0}^{\infty} \bar{g}|\beta-\bar{\beta}|\left|g-g_{0}\right|\left|\frac{\partial \bar{h}_{0}}{\partial r}\right| d r \\
& +\int_{0}^{\infty} \bar{g}|\beta-\bar{\beta}|\left|\bar{g}-\bar{g}_{0}\right|\left|\frac{\partial h_{0}}{\partial r}-2 \frac{\partial \bar{h}_{0}}{\partial r}\right| d r+\int_{0}^{\infty} \bar{g}|\beta-\bar{\beta}| \frac{|\eta|}{r} d r .
\end{aligned}
$$

By Lemma 2 and (3.7) the first integral on the right is bounded by a constant multiple of:

$$
\int_{0}^{\infty} \bar{\alpha}|\beta-\bar{\beta}|(1-\alpha)^{1 / 2} d r
$$

which in turn is bounded by (see (3.11)):

$$
\left(\int_{0}^{\infty} \bar{\alpha}^{2}(\beta-\bar{\beta})^{2} d r\right)^{1 / 2}\left(\int_{0}^{\infty}(1-\alpha) d r\right)^{1 / 2} \leqq\left(\frac{N}{4 \pi}\right)^{1 / 2} N^{1 / 2}=\frac{N}{2 \pi^{1 / 2}}
$$

To estimate the last two integrals in (4.2) we shall use the following: For any function $f \in L^{2}(0, \infty)$ we have $\bar{f} \in L^{2}(0, \infty)$ and

$$
\int_{0}^{\infty} \bar{f}^{2} d r \leqq 4 \int_{0}^{\infty} f^{2} d r
$$

This fact is seen as follows. Since $\partial \bar{f} / \partial r=(f-\bar{f}) / r$ and since

$$
r \bar{f}^{2}(r)=\frac{1}{r}\left(\int_{0}^{r} f d r\right)^{2} \leqq \int_{0}^{r} f^{2} d r \rightarrow 0 \quad \text { as } \quad r \rightarrow 0 .
$$

we have:

$$
\int_{0}^{r} \bar{f}^{2} d r=r \bar{f}^{2}-\int_{0}^{r} r \cdot 2 \bar{f} \frac{(f-\bar{f})}{r} d r
$$


that its:

$$
\int_{0}^{r} \bar{f}^{2} d r=\int_{0}^{r} 2 f \bar{f} d r-r \bar{f}^{2} \leqq \int_{0}^{r} 2 f \bar{f} d r \leqq 2\left(\int_{0}^{r} f^{2} d r\right)^{1 / 2}\left(\int_{0}^{r} \bar{f}^{2} d r\right)^{1 / 2} .
$$

Therefore,

$$
\int_{0}^{r} \bar{f}^{2} d r \leqq 4 \int_{0}^{r} f^{2} d r
$$

holds for every $r$, from which the result follows. Setting now

$$
\gamma:=(1-\alpha)^{1 / 2}
$$

we have by Lemma 2 :

$$
\left|\bar{g}-\bar{g}_{0}\right| \leqq \int_{0}^{r}\left|g-g_{0}\right| d r \leqq c \bar{\gamma}
$$

Thus, the second intergal on the right in (4.2) is bounded by a constant multiple of

$$
\int_{0}^{\infty} \bar{\alpha}|\beta-\bar{\beta}| \bar{\gamma} d r
$$

which by (4.3) is in turn bounded by:

$$
\left(\int_{0}^{\infty} \bar{\alpha}^{2}(\beta-\bar{\beta})^{2} d r\right)^{1 / 2}\left(\int_{0}^{\infty} \bar{\gamma}^{2} d r\right)^{1 / 2} \leqq\left(\frac{N}{4 \pi}\right)^{1 / 2}\left(4 \int_{0}^{\infty} \gamma^{2} d r\right)^{1 / 2}=\frac{N}{\pi^{1 / 2}} .
$$

From (3.16) we obtain

$$
\frac{\eta}{r} \leqq \frac{C}{r} \int_{0}^{r}\left|\bar{g}-\bar{g}_{0}\right| d r \leqq C c \overline{\bar{\gamma}}
$$

Therefore, the third integral on the right in (4.2) is bounded by a constant multiple of

$$
\int_{0}^{\infty} \bar{\alpha}|\beta-\bar{\beta}| \bar{\gamma} d r
$$

which, applying (4.3) twice, is bounded by:

$$
\left(\int_{0}^{\infty} \bar{\alpha}^{2}(\beta-\bar{\beta})^{2} d r\right)^{1 / 2}\left(\int_{0}^{\infty} \bar{\gamma}^{2} d r\right)^{1 / 2} \leqq\left(\frac{N}{4 \pi}\right)^{1 / 2}\left(16 \int_{0}^{\infty} \gamma^{2} d r\right)^{1 / 2}=\frac{2 N}{\pi^{1 / 2}} .
$$

We conclude from the above that $I_{1}$ is bounded from above by a constant multiple of $N$ :

$$
I_{1} \leqq C N .
$$

We now turn to $I_{2}$, which, according to (3.22), is equal to $I_{2,1}+I_{2,2}$, where

$$
I_{2,1}=\int_{0}^{\infty}(1-\alpha) e^{\psi} g_{0} \frac{\partial \psi}{\partial u} d r
$$

and

$$
I_{2,2}=\int_{0}^{\infty}(1-\alpha) e^{\psi} \frac{\partial g_{0}}{\partial u} d r
$$


The quantity $\partial g_{0} / \partial u$ is uniformly bounded, $h_{0}$ being a classical solution. According to (3.6) $\psi$ is also uniformly bounded. Thus $I_{2,2}$ is bounded by a constant multiple of $N$. We are therefore left with $I_{2,1}$. The quantity $\partial \psi / \partial u$ is given by (3.25). The third term in (3.25) can be shown to be uniformly bounded. Therefore the contribution of this term to $I_{2,1}$ is bounded by a constant multiple of $N$. The contribution of the first term in (3.25) to $I_{2,1}$ is bounded by a constant multiple of

$$
\int_{0}^{\infty}(1-\alpha) \bar{\alpha}|\beta-\bar{\beta}| d r
$$

which, since $0 \leqq 1-\alpha \leqq 1$, is in turn bounded by $N / 2 \pi^{1 / 2}$. We are left with the contribution of the second term in (3.25). We have thus arrived at this point to the following conclusion:

$$
I_{2} \leqq C N+C \int_{0}^{\infty}(1-\alpha)\left(\int_{r}^{\infty}\left|\frac{\partial \bar{h}_{0}}{\partial r}\right| \frac{|\theta|}{r} d r\right) d r .
$$

Since the integral

$$
\int_{1}^{\infty}\left|\partial \bar{h}_{0} / \partial r\right| d r / r
$$

is uniformly bounded, for $r \geqq 1$ we have

$$
\int_{r}^{\infty}\left|\frac{\partial \bar{h}_{0}}{\partial r}\right| \frac{|\theta|}{r} d r \leqq C \sup _{r}|\theta| .
$$

Also, since $\partial \bar{h}_{0} / \partial r$ is uniformly bounded, for $r<1$ we have

$$
\begin{aligned}
& \int_{r}^{\infty}\left|\frac{\partial \bar{h}_{0}}{\partial r}\right| \frac{|\theta|}{r} d r=\int_{r}^{1}\left|\frac{\partial \bar{h}_{0}}{\partial r}\right| \frac{|\theta|}{r} d r+\int_{1}^{\infty}\left|\frac{\partial \bar{h}_{0}}{\partial r}\right| \frac{|\theta|}{r} d r \\
& \leqq C\left[\left(\int_{0}^{1} \frac{\theta^{2}}{r} d r\right)^{1 / 2}\left(\int_{r}^{1} \frac{d r}{r}\right)^{1 / 2}+\sup _{r}|\theta|\right] \\
& \leqq C H(u)\left[\log ^{1 / 2}(1 / r)+1\right],
\end{aligned}
$$

where

$$
H:=\left(\int_{0}^{1} \frac{\theta^{2}}{r} d r\right)^{1 / 2}+\sup _{r}|\theta| .
$$

Since $\theta / r^{1 / 2} \in L^{2}\left(0, u_{0}\right) \times(0,1)$ and

$$
\sup _{r}|\theta| \in L^{2}\left(0, u_{0}\right)
$$

the function $H(u)$ belongs to $L^{2}\left(0, u_{0}\right)$. We conclude from the above that:

$$
\begin{aligned}
\int_{0}^{\infty}(1-\alpha)\left(\int_{r}^{\infty}\left|\frac{\partial h_{0}}{\partial r}\right| \frac{|\theta|}{r} d r\right) d r \\
\quad \leqq C H\left\{\int_{0}^{1}(1-\alpha) \log ^{1 / 2}(1 / r) d r+\int_{0}^{\infty}(1-\alpha) d r\right\} .
\end{aligned}
$$

We shall now show: 
Lemma 3. There is a universal constant $c$ such that

$$
\int_{0}^{1}(1-\alpha) \log ^{1 / 2}(1 / r) d r \leqq c N\left(1+\log \left(1+\frac{1}{N}\right)\right) .
$$

Proof. Let us recall the following [5]: If $\Omega$ is a domain in $\mid R^{n}$ and $A$ is an $N$-function, the Orlicz space $L_{A}(\Omega)$ is defined to be the linear hull of the set of all measureable functions $f$ defined on $\Omega$ and such that

$$
\int_{\Omega} A(|f(x)|) d x<\infty
$$

it is a Banach space with respect to the norm

$$
\|f\|_{A, \Omega}=\inf \left\{k>0: \int_{\Omega} A\left(\frac{|f(x)|}{k}\right) d x \leqq 1\right\} .
$$

If $A$ and $\widetilde{A}$ are complementary $N$-functions, the following inequality holds:

$$
\left|\int_{\Omega} f_{1}(x) f_{2}(x) d x\right| \leqq 2\left\|f_{1}\right\|_{A, \Omega}\left\|f_{2}\right\|_{\tilde{A}, \Omega}
$$

We shall apply the above to the case where $\Omega=(0,1), A$ and $\tilde{A}$ are the complementary $N$-functions

$$
A(t)=(1+t) \log (1+t)-t
$$

and

$$
\tilde{A}(t)=e^{t}-t-1,
$$

and $f_{1}=1-\alpha$ and $f_{2}=\log ^{1 / 2}(1 / r)$. Then

$$
\int_{0}^{1}(1-\alpha) \log ^{1 / 2}(1 / r) d r \leqq 2\|(1-\alpha)\|_{A,(0,1)}\left\|\log ^{1 / 2}(1 / r)\right\|_{\tilde{A},(0,1)}
$$

We have

$$
\begin{gathered}
\int_{0}^{1} A\left(\log ^{1 / 2}(1 / r)\right) d r=\int_{0}^{1}\left(e^{\log ^{1 / 2}}(1 / r)-\log ^{1 / 2}(1 / r)-1\right) d r \\
=\int_{0}^{\infty}\left(e^{s^{1 / 2}}-s^{1 / 2}-1\right) e^{-s} d s=c,
\end{gathered}
$$

where $c$ is a finite constant. Hence

$$
\left\|\log ^{1 / 2}(1 / r)\right\|_{\tilde{A},(0,1)} \leqq c .
$$

Now,

$$
\|(1-\alpha)\|_{A,(0,1)} \leqq\|(1-\alpha)\|_{A,(0, \infty)},
$$

and

$\|(1-\alpha)\|_{A,(0, \infty)}=\inf \left\{k>0: \int_{0}^{\infty}\left[\left(1+\frac{(1-\alpha)}{k}\right) \log \left(1+\frac{(1-\alpha)}{k}\right)-\frac{(1-\alpha)}{k}\right] d r \leqq 1\right\}$. 
For $t \geqq 0$ it holds:

$$
(1+t) \log (1+t)-t \leqq \log (1+t)
$$

Thus, taking into account the fact that $(1-\alpha) \leqq 1$, we obtain:

$$
\begin{aligned}
& \int_{0}^{\infty}\left\{\left(1+\frac{(1-\alpha)}{k}\right) \log \left(1+\frac{(1-\alpha)}{k}\right)-\frac{(1-\alpha)}{k}\right] d r \\
& \leqq \int_{0}^{\infty} \frac{(1-\alpha)}{k} \log \left(1+\frac{(1-\alpha)}{k}\right) d r \\
& \leqq \int_{0}^{\infty} \frac{1-\alpha}{k} \log \left(1+\frac{1}{k}\right) d r=\frac{N}{f(k)} .
\end{aligned}
$$

where

$$
f(k):=\frac{k}{\log \left(1+\frac{1}{k}\right)} .
$$

We conclude that if for a certain positive value $k_{1}$ of $k f\left(k_{1}\right) \geqq N$, then $\|(1-\alpha)\|_{A,(0, \infty)}$ $\leqq k_{1}$. Let

$$
k_{1}:=N\left(1+\log \left(1+\frac{1}{N}\right)\right)
$$

Then

$$
f\left(k_{1}\right)=N \cdot \frac{\left(1+\log \left(1+\frac{1}{N}\right)\right)}{\log \left[1+\frac{1}{N\left(1+\log \left(1+\frac{1}{N}\right)\right)}\right]}>N
$$

therefore:

$$
\|(1-\alpha)\|_{A,(0, \infty)} \leqq k_{1} .
$$

Considering (4.13), (4.12) and (4.11), the lemma follows from (4.10).

From (4.4), (4.7), (4.9) and Lemma 3, together with (3.20) we conclude that:

$$
\frac{\partial L}{\partial u} \leqq C_{1} N+C_{2} H N+C_{3} H N\left(1+\log \left(1+\frac{1}{N}\right)\right) .
$$

Thus, taking into account the facts that (see (3.10) and (3.12)) $N / k \leqq k N$ and $L \leqq C M_{0}$, we conclude:

$$
\frac{\partial L}{\partial u} \leqq C^{\prime}(1+H) L \log \left(\frac{2 C M_{0}}{L}\right)
$$


Let us set:

$$
x:=L / 2 C M_{0},
$$

since $L(u)$ is an absolutely continuous function of $u$ in $\left[0, u_{0}\right]$, the same is true for $x(u)$, and we have $0 \leqq x \leqq 1 / 2$. According to (4.15) $x$ satisfies the ordinary differential inequality:

$$
\frac{d x}{d u} \leqq \operatorname{ax} \log \left(\frac{1}{x}\right)
$$

where:

$$
a:=C^{\prime}(1+H) \text {. }
$$

Since $H \in L^{2}\left(0, u_{0}\right)$, the function $a(u)$ also belongs to $L^{2}\left(0, u_{0}\right)$. Integrating (4.17) we obtain:

$$
\log (1 / \log (1 / x(u)))-\log (1 / \log (1 / x(0))) \leqq \int_{0}^{u} a d u
$$

which yields

$$
x(u) \leqq(x(0))^{e^{-\stackrel{u}{-} a d u}}
$$

The exponent of $x(0)$ in the above inequality is less than 1 and decreases monotonically with $u$. But since $a \xi L^{2}\left(0, u_{0}\right)$, and therefore a fortiori $a \in L^{2}\left(0, u_{0}\right)$, this exponent is greater than 0 . Hence $\dot{x}(0)=0$ implies $x(u)=0$ for all $u \in\left[0, u_{0}\right]$. Now, the fact that the initial data of $h$ and $h_{0}$ coincide implies that $L(0)=0$. Thus, in fact, $x(0)=0$, and therefore $L(u)=0$ for all $u \in\left[0, u_{0}\right]$. It follows that $\alpha=1$ for all $(u, r) \in\left[0, u_{0}\right] \times[0, \infty]$. Hence, $\beta-\bar{\beta}=0$, that is, $\partial \bar{\beta} / \partial r=0$, for all $r$ and all $u \in\left[0, u_{0}\right]$. Since at each $u \bar{\beta} \rightarrow 0$ as $r \rightarrow \infty$, we conclude that $\bar{\beta}=0$, hence $\beta=0$. Therefore $h=h_{0}$ on $\left[0, u_{0}\right] \times[0, \infty[$, and the proof of Theorem 1 is now complete.

We note that the generalized solution may not be unique beyond the domain of existence of the classical solution as there may be bifurcations across the singular cones.

\section{References}

1. Christodoulou, D.: The problem of a self-gravitating scalar field. Commun. Math. Phys. 105, 337 (1986)

2. Christodoulou, D.: Global existence of generalized solutions of the spherically symmetric Einsteinscalar equations in the large. Commun. Math. Phys. 106, 587 (1986)

3. Leray, J.: Sur le mouvement d'un liquide visquex emplissant l'espace. Acta Mah. 63, 193-248 (1934)

4. Caffarelli, L., Kohn, R., Nirenberg, L.: Partial regularity of suitable weak solutions of the NavierStokes equations. Commun. Pure Appl. Math., Vol. XXXV, 771-831 (1982)

5. Kranosel'skii, M. A., Rutickii, Ya. B.: Convex functions and Orlicz spaces. Groningen, The Netherlands: Noordhoff 1961

Communicated by S.-T. Yau

Received November 9, 1984; in revised form August 29, 1986 
